\title{
Synthesis, heteronuclear NMR and X-ray crystallographic studies of two dinuclear diorganotin(IV) dithiocarbamate macrocycles
}

\author{
Reyna Reyes-Martínez, Rocio Mejia-Huicochea, Jorge A. Guerrero-Alvarez, \\ Herbert Höpfl, and Hugo Tlahuext*
}

Centro de Investigaciones Químicas, Universidad Autónoma del Estado de Morelos, Av. Universidad 1001, Col. Chamilpa, C.P. 62209, Cuernavaca, Morelos, Mexico

E-mail:tlahuext@,ciq.uaem.mx

Dedicated to Professor Rosalinda Contreras on occasion of her $60^{\text {th }}$ birthday

\begin{abstract}
Two new dinuclear diorganotin(IV) dithiocarbamate (dtc) macrocycles, $\left[\mathrm{Me}_{2} \mathrm{Sn}(\mathrm{dtc})\right]_{2}(\mathbf{1})$ and $\left[n \mathrm{Bu}_{2} \mathrm{Sn}(\mathrm{dtc})\right]_{2}$ (2), have been synthesized from $\mathrm{R}_{2} \mathrm{SnCl}_{2}(\mathrm{R}=\mathrm{Me}, n \mathrm{Bu})$ and the bis(dithiocarbamate) derivative of $N, N^{\prime}$-dibenzylhexamethylene-1,6-diamine. These complexes have been characterized by elemental analysis, FAB mass spectrometry, IR and NMR $\left({ }^{1} \mathrm{H},{ }^{13} \mathrm{C}\right.$, ${ }^{119} \mathrm{Sn}$ ) spectroscopy, as well as single crystal X-ray crystallography.

The dtc ligands are coordinated to the tin atoms in the anisobidentate manner, both in solution and the solid state. The metal centers are hexacoordinated and the coordination polyhedron of the tin atoms can be described as bicapped tetrahedron or skew trapezoidal bipyramid. The cavities of the 26-membered macrocycles are hydrophobic with four sulfur atoms directed into the interior and have an inner diameter of approximately $4.1 \times 5.6 \AA^{2}$. The crystal structure of $\left[\mathrm{Me}_{2} \mathrm{Sn}(\mathrm{dtc})\right]_{2}(\mathbf{1})$ is stabilized by the presence of two chloroform molecules per asymmetric unit, which participate in intermolecular $\mathrm{C}-\mathrm{H} \cdots \mathrm{S}, \mathrm{C}-\mathrm{H} \cdots \mathrm{Cl}$ hydrogen bonding and $\mathrm{S} \cdots \mathrm{Cl}$ donor acceptor interactions. On the other hand, the crystal structure of $\left[n \mathrm{Bu}_{2} \mathrm{Sn}(\mathrm{dtc}]_{2}(2)\right.$ is stabilized additionally by intermolecular $\mathrm{C}-\mathrm{H} \cdots \pi$ interactions.
\end{abstract}

Keywords: Organotin compounds, dithiocarbamate ligands, macrocycles, self-assembly, ${ }^{119} \mathrm{Sn}$ NMR

\section{Introduction}

During the last few years the metal ion directed self assembly of ligands to well-defined structures has been exploited mainly in two directions: first, the generation of discrete cyclooligomeric molecular structures having cavities and, second, the creation of microporous 
coordination polymers. ${ }^{1}$ For this purpose mainly transition metals and easily available ligands such as carboxylic acids and amines have been applied. The number of reports on systems containing representative elements or organometallic building blocks such as organotin moieties is relatively small so far. ${ }^{2,3}$ The same is true for the application of dithiocarbamate (dtc) ligands in supramolecular chemistry. Moreover, metallomacrocycles based on dtc ligands are almost unexplored. ${ }^{4}$

Recently, synthetic strategies have been developed for the preparation of macrocyclic and microporous materials based on diorganotin dicarboxylates. ${ }^{5}$ In this contribution we present an extension of this strategy to the synthesis of diorganotin(IV) dithiocarbamate macrocycles, the results of which are presented herein.

\section{Results and Discussion}

\section{Synthetic Methods}

The secondary diamine, $N, N^{\prime}$-dibenzylhexamethylene-1,6-diamine, used as starting material for the preparation of the diorganotin complexes, was obtained in $90 \%$ yield by condensation of benzaldehyde with 1,6-hexamethylenediamine in ethanol followed by reduction of the Schiff base with $\mathrm{NaBH}_{4}$ in methanol. ${ }^{6}$ One-pot syntheses from this amine, carbon disulfide, triethylamine and $\mathrm{R}_{2} \mathrm{SnCl}_{2}(\mathrm{R}=\mathrm{Me}, n \mathrm{Bu})$ gave colorless precipitates that were recrystallized from chloroform and a mixture of dichloromethane/methanol to give the dinuclear diorganotin(IV) dithiocarbamate macrocycles, $\left[\mathrm{Me}_{2} \mathrm{Sn}(\mathrm{dtc})\right]_{2}(\mathbf{1})$ and $\left[n \mathrm{Bu}_{2} \mathrm{Sn}(\mathrm{dtc}]_{2}(\mathbf{2})\right.$, in yields of 54 and $36 \%$, respectively (Scheme 1). These products are soluble in non polar solvents such as dichloromethane and chloroform, but insoluble in polar solvents such as acetone, ethanol, methanol, dimethyl sulfoxide, acetonitrile and water.

The composition and molecular structures of compounds $\mathbf{1}$ and $\mathbf{2}$ were established by elemental analysis, mass spectrometry, IR and NMR $\left({ }^{1} \mathrm{H},{ }^{13} \mathrm{C},{ }^{119} \mathrm{Sn}\right)$ spectroscopy, as well as single crystal X-ray crystallography. 


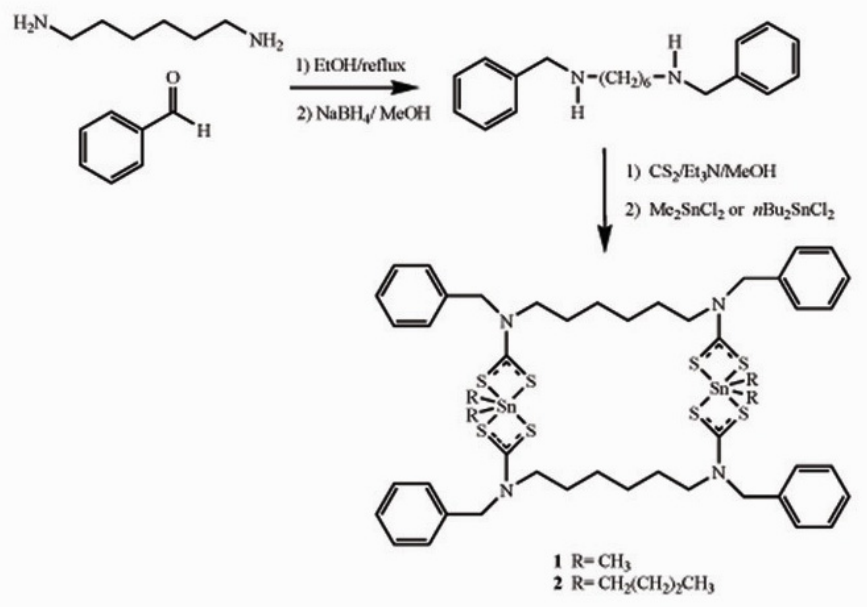

Scheme 1. Preparation of the dinuclear diorganotin(IV) dithiocarbamate macrocycles, $\left[\mathrm{Me}_{2} \mathrm{Sn}(\mathrm{dtc})\right]_{2}(\mathbf{1})$ and $\left[n \mathrm{Bu}_{2} \mathrm{Sn}(\mathrm{dtc}]_{2}(\mathbf{2})\right.$.

\section{Spectroscopic characterization}

The IR spectra of $\mathbf{1}$ and $\mathbf{2}$ gave evidence for the formation of the dithiocarbamate functions and their coordination to the diorganotin fragments. The bands resulting from the stretching vibrations of the $\mathrm{C}-\mathrm{N}$ bonds at 1476 and $1475 \mathrm{~cm}^{-1}$ have wavenumbers that are intermediate when compared to those reported for $\mathrm{C}-\mathrm{N}$ single bonds $\left(1250-1360 \mathrm{~cm}^{-1}\right)$ and $\mathrm{C}=\mathrm{N}$ double bonds (1640-1690 $\mathrm{cm}^{-1}$ ), suggesting partial double bond character and, therefore, partial delocalization of $\pi$-electron density within the dithiocarbamate functions. ${ }^{7}$ For the $\mathrm{CS}_{2}{ }^{-}$groups two bands were observed, $v\left(\mathrm{CS}_{2}\right)_{\text {as }}$ and $v\left(\mathrm{CS}_{2}\right)_{\mathrm{s}}\left(1239,981 \mathrm{~cm}^{-1}\right.$ for $\mathbf{1}$ and $1238,980 \mathrm{~cm}^{-1}$ for 2$)$, which are characteristic for an anisobidentate chelation mode of the ligands to the metal atoms. ${ }^{8}$

The ${ }^{1} \mathrm{H}$ and ${ }^{13} \mathrm{C}$ NMR spectra of compounds $\mathbf{1}$ and $\mathbf{2}$ were assigned completely by 2D correlation experiments (COSY and HSQC). A comparison of the ${ }^{1} \mathrm{H}$ NMR spectra between the $N, N^{\prime}$-dibenzylhexamethylene-1,6-diamine and the resulting products showed significant shift displacements to lower fields for the $\mathrm{NCH}_{2 \text { benzyl }}$ and $\mathrm{NCH}_{2 \text { chain }}$ methylene hydrogen atoms (1: $\Delta \delta$ $=1.39$ and $1.02 \mathrm{ppm}, 2 \mathrm{~s}: \Delta \delta=1.42$ and $1.07 \mathrm{ppm}$ ), thus indicating the formation of the dithiocarbamate complexes. For the corresponding carbon atoms shift displacements in the same direction were observed (1: $\Delta \delta=3.2$ and $4.4 \mathrm{ppm}, \mathbf{2}: \Delta \delta=3.2$ and $4.6 \mathrm{ppm}$ ). The $\mathrm{NCS}_{2}$ carbon atoms gave signals at $\delta=201.0$ and $201.8 \mathrm{ppm}$, respectively. Interestingly, in the ${ }^{1} \mathrm{H}$ NMR spectra all methylene hydrogen atoms gave rise to broad signals, indicating that the complexes are involved in at least one dynamic process. Considering the macrocyclic nature of the products (vide infra), it can be supposed that there exist conformational equilibria in solution.

The ${ }^{119} \mathrm{Sn}$ RMN spectroscopic data allowed to establish the coordination number of the tin atoms. According to the information reported in the literature, ${ }^{9}$ the ${ }^{119} \mathrm{Sn}$ NMR chemical shift values for compounds $1(\delta=-332 \mathrm{ppm})$ and $\mathbf{2}(\delta=-333 \mathrm{ppm})$ indicate that the tin atoms are 
hexa-coordinated in solution. For compound 1 it was possible to measure the ${ }^{2} J_{\text {Sn-H }}$ coupling constant in the ${ }^{1} \mathrm{H}$ NMR spectrum $(82 \mathrm{~Hz})$.

The assembly of the starting materials described in Scheme 1 can give either cyclooligomeric or polymeric products. The mass spectra $\left(\mathrm{FAB}^{+}\right)$of compounds $\mathbf{1}$ and $\mathbf{2}$ support the assumption that dimeric structures had been obtained, since in both cases peaks were only observed in the range corresponding to substances having a dimeric composition. However, the peak corresponding to the molecular ion could be detected only in the case of compound $\mathbf{1}, \mathrm{m} / \mathrm{z} 1190$, while for $\mathbf{2}$ only fragments derived from a dimeric structure could be observed. In this case, one of the most prominent peaks, $m / z 1066$, corresponds to a dinuclear species, which has suffered loss of one of the $N, N^{\prime}$-dibenzylhexamethylene-1,6-diamine fragments (see Experimental Section).

\section{X-Ray crystallographic study}

The recrystallization processes described above for compounds $\mathbf{1}$ and $\mathbf{2}$ gave also crystals that were suitable for X-ray crystallographic studies. Perspective views of the molecular geometries (top and lateral views) are shown in Figures 1a-1d. The most relevant crystallographic data for $\mathbf{1}$ and $\mathbf{2}$ have been summarized in Table 1. Selected bond lengths, bond angles and torsion angles are outlined in Table 2.

a)

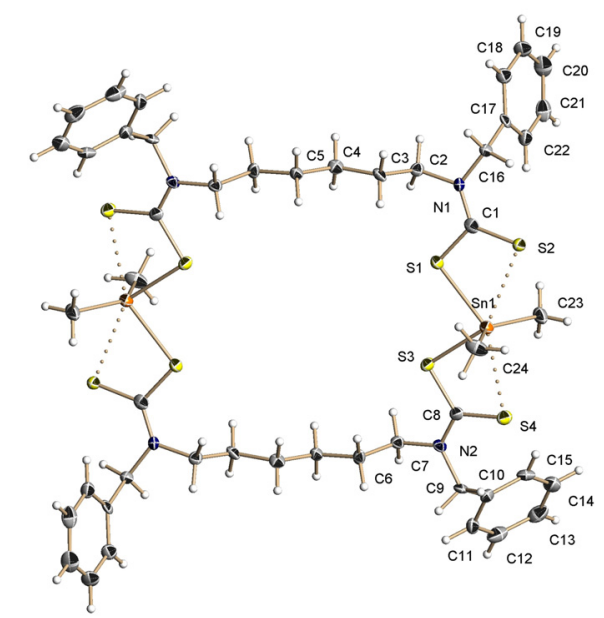

b)

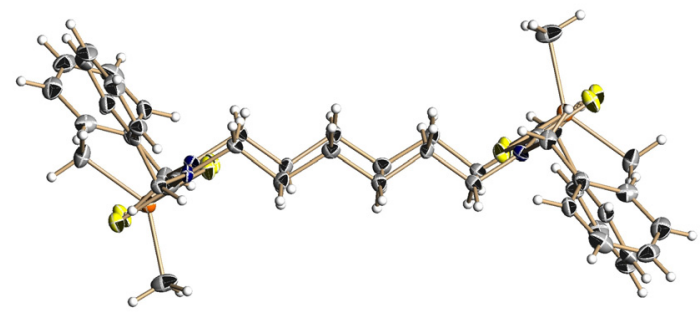

c)

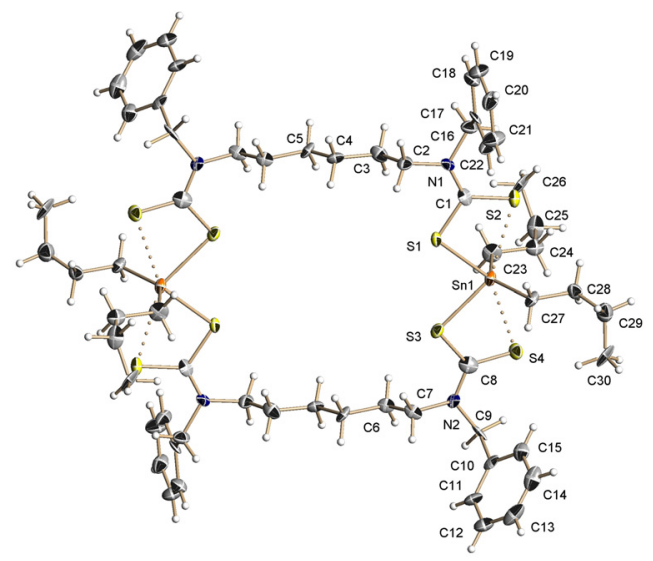

d)

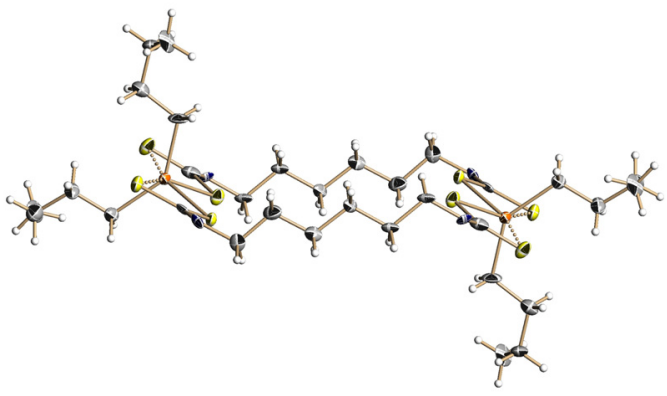

Figure 1. Perspective views (top and lateral) of the molecular structures of a), b) $\left[\mathrm{Me}_{2} \mathrm{Sn}(\mathrm{dtc})\right]_{2}$ (1) and c), d) $\left[n \mathrm{Bu}_{2} \mathrm{Sn}(\mathrm{dtc})\right]_{2}$ (2). The benzyl groups have been omitted for clarity in b) and d). 
Table 1. Crystallographic data for compounds 1 and $\mathbf{2}$

\begin{tabular}{|c|c|c|}
\hline Crystal data & {$\left[\mathrm{Me}_{2} \mathrm{Sn}(\mathrm{dtc})\right]_{2}$} & {$\left[n \mathrm{Bu}_{2} \mathrm{Sn}(\mathrm{dtc})\right]_{2}$} \\
\hline Formula & $\mathrm{C}_{50} \mathrm{H}_{66} \mathrm{Cl}_{6} \mathrm{~N}_{4} \mathrm{~S}_{8} \mathrm{Sn}_{2}$ & $\mathrm{C}_{61} \mathrm{H}_{90} \mathrm{Cl}_{2} \mathrm{~N}_{4} \mathrm{~S}_{8} \mathrm{Sn}_{2}$ \\
\hline Crystal size (mm) & $0.15 \times 0.21 \times 0.36$ & $0.17 \times 0.23 \times 0.38$ \\
\hline $\mathrm{MW}\left(\mathrm{g} \mathrm{mol}^{-1}\right)$ & 1429.63 & 1444.13 \\
\hline \multirow[t]{2}{*}{ Space group } & $\mathrm{P} 2(1) / \mathrm{c}$ & $\mathrm{C} 2 / \mathrm{c}$ \\
\hline & Cell Parameters & \\
\hline$a(\AA)$ & $18.505(4)$ & $41.731(8)$ \\
\hline$b(\AA)$ & $10.912(2)$ & $10.372(2)$ \\
\hline$c(\AA)$ & $15.712(3)$ & $16.951(3)$ \\
\hline$\alpha\left({ }^{\circ}\right)$ & 90.0 & 90.0 \\
\hline$\beta\left({ }^{\circ}\right)$ & $96.51(3)$ & $110.30(3)$ \\
\hline$\gamma\left({ }^{\circ}\right)$ & 90.0 & 90.0 \\
\hline $\mathrm{V}\left(\AA^{3}\right)$ & $3152.1(11)$ & $6881(2)$ \\
\hline Z & 2 & 4 \\
\hline$\mu\left(\mathrm{mm}^{-1}\right)$ & 1.348 & 1.085 \\
\hline \multirow[t]{2}{*}{$\rho_{\text {calcd }}\left(\mathrm{g} \mathrm{cm}^{-3}\right)$} & 1.506 & 1.394 \\
\hline & Data Collection & \\
\hline$\theta$ limits $(\AA)$ & $2.17<\theta<25.00^{\circ}$ & $2.03<\theta<25.00$ \\
\hline hkl limits & $-17,21 ;-12,12 ;-18,18$ & $-49,41 ;-12,12 ;-13,20$ \\
\hline no. Collected refl. & 14940 & 16248 \\
\hline \multirow[t]{2}{*}{ No. Ind refl. $\left(\mathrm{R}_{\text {int }}\right)$} & $5314(0.0626)$ & $6036(0.1290)$ \\
\hline & Refinement & \\
\hline $\mathrm{R}$ & 0.0580 & 0.1202 \\
\hline $\mathrm{R}_{\mathrm{w}}$ & 0.1068 & 0.1800 \\
\hline No. Of variables & 318 & 338 \\
\hline GOF & 1.098 & 1.232 \\
\hline$\Delta \rho_{\min }\left(\mathrm{e} \AA^{-3}\right)$ & -831 & -1.727 \\
\hline$\Delta \rho_{\max }\left(\mathrm{e} \AA^{-3}\right)$ & 873 & 1.154 \\
\hline
\end{tabular}

The dinuclear tin complexes have centrosymmetric structures characterized by $26-$ membered $\mathrm{C}_{16} \mathrm{~N}_{4} \mathrm{~S}_{4} \mathrm{Sn}_{2}$ ring systems, in which four sulfur atoms are directed into the interior of the cavity. The dtc moieties are coordinated to the tin atoms in the anisobidentate manner that is typical for this type of ligands. The covalent Sn-S bonds range from 2.516(3) to 2.532(3) $\AA$, and the secondary interactions from $2.885(2)$ to $2.958(4) \AA$ (Table 2). The metal centers are hexacoordinated and the coordination polyhedron of the tin atoms can be described as a bicapped tetrahedron or skew trapezoidal bipyramid. ${ }^{10}$ The four atoms comprising the tetrahedron (S1, S3, $\mathrm{C} 23$ and C24) form four angles typical for tetrahedral structures with the tin atoms, 102.7(4) 109.0(3) $)^{\circ}$, and two angles that show the influence of the capping atoms, $\mathrm{S}(3)-\mathrm{Sn}(1)-\mathrm{S}(1)=$ 
82.82(5), 83.31(10 $)^{\mathrm{o}}$ and $\mathrm{C}(24)-\mathrm{Sn}(1)-\mathrm{C}(23)=137.7(3), 137.8(5)^{\mathrm{o}}$. The dimensions of the hydrophobic cavity of the macrocycle are $4.1 \times 5.6 \AA^{2}$ (Figure 2 ). The transannular S ...S and C...C distances are 7.66, 9.41 $\AA$ and 7.93, 9.17 $\AA$ for $\mathbf{1}$ and $\mathbf{2}$, respectively.

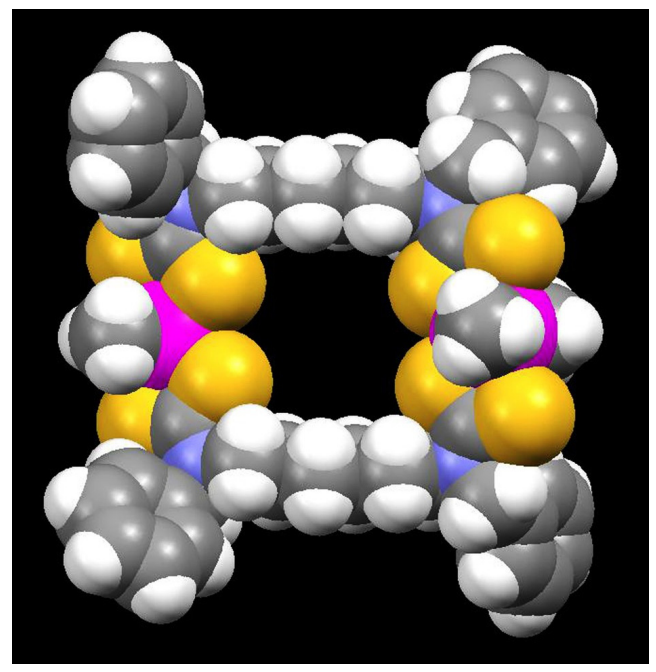

Figure 2. Spacefiiling model of compound 1 showing the macrocyclic cavity $\left(4.1 \times 5.6 \AA^{2}\right)$.

The supramolecular structure in the crystal lattices of compound $\mathbf{1}$ is stabilized by the presence of two chloroform molecules per asymmetric unit, which participate in $\mathrm{C}-\mathrm{H} \cdots \mathrm{S}$ and $\mathrm{C}$ $\mathrm{H} \cdots \mathrm{Cl}$ hydrogen bonds ${ }^{11}$ and $\mathrm{S} \cdots \mathrm{Cl}$ donor acceptor interactions. ${ }^{12}$ The interatomic $\mathrm{S} \cdots \mathrm{Cl}$ distances have a value of $3.373 \AA$, which is significantly shorter than the corresponding sum of the van der Waals radii $\left[\Sigma \mathrm{r}_{\mathrm{vdw}}(\mathrm{S}, \mathrm{Cl})=3.70 \AA\right]$ (Figure 3$)$.

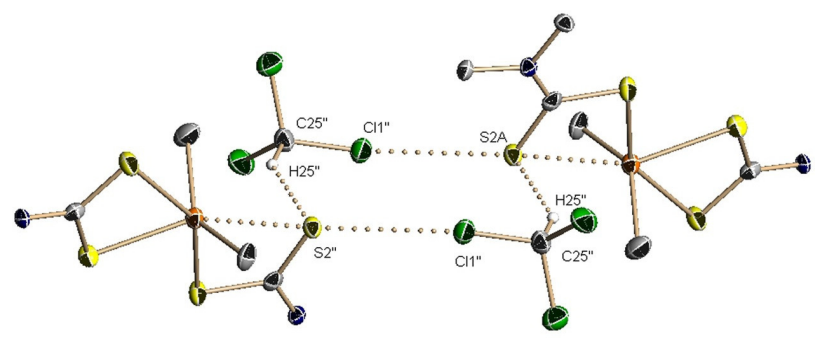

Figure 3. Fragment of the crystal lattice of $\left[\mathrm{Me}_{2} \mathrm{Sn}(\mathrm{dtc})\right]_{2}(\mathbf{1})$ showing the $\mathrm{S} \cdots \mathrm{Cl}$ donor acceptor interactions.

On the other hand, the crystal lattice of $\left[n \mathrm{Bu}_{2} \mathrm{Sn}(\mathrm{dtc})\right]_{2}(2)$ is stabilized additionally by C$\mathrm{H} \cdots \pi$ interactions $(\mathrm{C}-\mathrm{H} \cdots \mathrm{Cg}=3.24 \AA$ ) between adjacent benzyl moieties (Figure 4). 


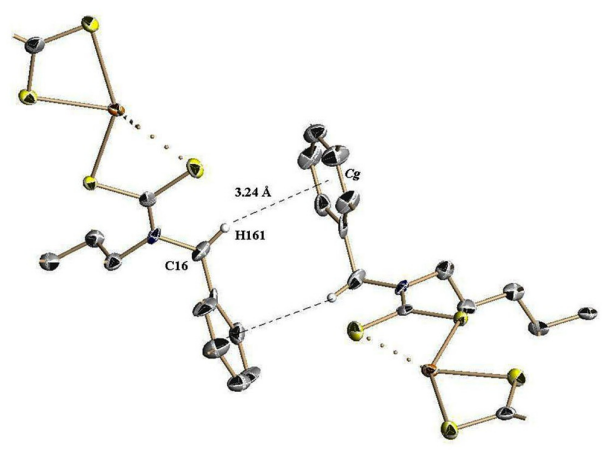

Figure 4. Fragment of the crystal lattice of $\left[n \mathrm{Bu}_{2} \mathrm{Sn}(\mathrm{dtc})\right]_{2}(\mathbf{2})$ showing the $\mathrm{C}-\mathrm{H} \cdots \pi$ interactions.

Table 2. Selected bond lengths, bond angles and torsion angles for compounds $\mathbf{1}$ and $\mathbf{2}$

\begin{tabular}{|c|c|c|c|}
\hline \multicolumn{2}{|c|}{1} & \multicolumn{2}{|c|}{2} \\
\hline \multicolumn{4}{|c|}{ Bond Lengths $(\AA)$} \\
\hline $\operatorname{Sn}(1)-C(24)$ & $2.115(6)$ & $\operatorname{Sn}(1)-C(23)$ & $2.082(11)$ \\
\hline $\operatorname{Sn}(1)-C(23)$ & $2.121(6)$ & $\operatorname{Sn}(1)-C(27)$ & $2.143(11)$ \\
\hline $\operatorname{Sn}(1)-S(3)$ & $2.517(2)$ & $\operatorname{Sn}(1)-S(3)$ & $2.516(3)$ \\
\hline $\operatorname{Sn}(1)-S(1)$ & $2.521(2)$ & $\operatorname{Sn}(1)-S(1)$ & $2.531(3)$ \\
\hline $\operatorname{Sn}(1)-S(4)$ & $2.885(2)$ & $\operatorname{Sn}(1)-S(4)$ & $2.925(3)$ \\
\hline $\operatorname{Sn}(1)-S(2)$ & $2.995(2)$ & $\operatorname{Sn}(1)-S(2)$ & $2.957(4)$ \\
\hline $\mathrm{S}(1)-\mathrm{C}(1)$ & $1.752(7)$ & $\mathrm{S}(1)-\mathrm{C}(1)$ & $1.746(12)$ \\
\hline $\mathrm{S}(2)-\mathrm{C}(1)$ & $1.702(6)$ & $\mathrm{S}(2)-\mathrm{C}(1)$ & $1.718(13)$ \\
\hline $\mathrm{S}(3)-\mathrm{C}(8)$ & $1.755(6)$ & $\mathrm{S}(3)-\mathrm{C}(8)$ & $1.748(14)$ \\
\hline$S(4)-C(8)$ & $1.689(6)$ & $\mathrm{S}(4)-\mathrm{C}(8)$ & $1.700(14)$ \\
\hline $\mathrm{N}(1)-\mathrm{C}(1)$ & $1.323(7)$ & $\mathrm{N}(1)-\mathrm{C}(1)$ & $1.318(15)$ \\
\hline $\mathrm{N}(1)-\mathrm{C}(2)$ & $1.464(7)$ & $\mathrm{N}(1)-\mathrm{C}(2)$ & $1.488(14)$ \\
\hline $\mathrm{N}(2)-\mathrm{C}(8)$ & $1.329(7)$ & $\mathrm{N}(2)-\mathrm{C}(8)$ & $1.330(15)$ \\
\hline $\mathrm{N}(2)-\mathrm{C}(9)$ & $1.474(7)$ & $\mathrm{N}(2)-\mathrm{C}(9)$ & $1.472(14)$ \\
\hline \multicolumn{4}{|c|}{ Bond Angles (deg) } \\
\hline $\mathrm{C}(24)-\mathrm{Sn}(1)-\mathrm{C}(23)$ & $137.7(3)$ & $\mathrm{C}(23)-\mathrm{Sn}(1)-\mathrm{C}(27)$ & $137.8(5)$ \\
\hline $\mathrm{C}(24)-\mathrm{Sn}(1)-\mathrm{S}(3)$ & $105.59(19)$ & $\mathrm{C}(23)-\mathrm{Sn}(1)-\mathrm{S}(3)$ & $104.1(4)$ \\
\hline$C(23)-\operatorname{Sn}(1)-S(3)$ & $107.55(19)$ & $C(27)-\operatorname{Sn}(1)-S(3)$ & $106.5(3)$ \\
\hline $\mathrm{C}(24)-\mathrm{Sn}(1)-\mathrm{S}(1)$ & $104.42(19)$ & $C(23)-\operatorname{Sn}(1)-S(1)$ & $102.7(4)$ \\
\hline $\mathrm{C}(23)-\mathrm{Sn}(1)-\mathrm{S}(1)$ & $105.15(18)$ & $C(27)-\operatorname{Sn}(1)-S(1)$ & 109.0(3) \\
\hline$S(3)-\operatorname{Sn}(1)-S(1)$ & $82.82(5)$ & $S(3)-\operatorname{Sn}(1)-S(1)$ & $83.31(10)$ \\
\hline$C(24)-\operatorname{Sn}(1)-S(4)$ & $85.98(18)$ & $C(23)-\operatorname{Sn}(1)-S(4)$ & $85.5(4)$ \\
\hline$C(23)-\operatorname{Sn}(1)-S(4)$ & $84.15(18)$ & $\mathrm{C}(27)-\mathrm{Sn}(1)-\mathrm{S}(4)$ & $81.4(3)$ \\
\hline $\mathrm{S}(3)-\mathrm{Sn}(1)-\mathrm{S}(4)$ & $66.01(5)$ & $\mathrm{S}(3)-\mathrm{Sn}(1)-\mathrm{S}(4)$ & $65.70(10)$ \\
\hline$S(1)-\operatorname{Sn}(1)-S(4)$ & $148.80(5)$ & $S(1)-S n(1)-S(4)$ & $149.02(10)$ \\
\hline$C(24)-\operatorname{Sn}(1)-S(2)$ & $84.69(18)$ & $C(23)-\operatorname{Sn}(1)-S(2)$ & $83.7(4)$ \\
\hline $\mathrm{C}(23)-\mathrm{Sn}(1)-\mathrm{S}(2)$ & $81.52(18)$ & $\mathrm{C}(27)-\mathrm{Sn}(1)-\mathrm{S}(2)$ & $85.0(3)$ \\
\hline
\end{tabular}




\begin{tabular}{|c|c|c|c|}
\hline $\mathrm{S}(3)-\mathrm{Sn}(1)-\mathrm{S}(2)$ & $147.23(5)$ & $S(3)-S n(1)-S(2)$ & $148.60(10)$ \\
\hline $\mathrm{S}(1)-\mathrm{Sn}(1)-\mathrm{S}(2)$ & $64.43(5)$ & $S(1)-S n(1)-S(2)$ & $65.28(10)$ \\
\hline $\mathrm{S}(4)-\mathrm{Sn}(1)-\mathrm{S}(2)$ & $146.69(5)$ & $\mathrm{S}(4)-\mathrm{Sn}(1)-\mathrm{S}(2)$ & $145.70(10)$ \\
\hline$C(1)-S(1)-S n(1)$ & $95.8(2)$ & $C(1)-S(1)-\operatorname{Sn}(1)$ & $94.4(4)$ \\
\hline$C(1)-S(2)-\operatorname{Sn}(1)$ & $81.2(2)$ & $C(1)-S(2)-S n(1)$ & $81.1(4)$ \\
\hline $\mathrm{C}(8)-\mathrm{S}(3)-\mathrm{Sn}(1)$ & $93.0(2)$ & $C(8)-S(3)-\operatorname{Sn}(1)$ & $93.7(5)$ \\
\hline $\mathrm{C}(8)-\mathrm{S}(4)-\mathrm{Sn}(1)$ & $82.4(2)$ & $\mathrm{C}(8)-\mathrm{S}(4)-\mathrm{Sn}(1)$ & $81.4(5)$ \\
\hline $\mathrm{C}(1)-\mathrm{N}(1)-\mathrm{C}(16)$ & $122.8(5)$ & $C(1)-N(1)-C(16)$ & $122.2(10)$ \\
\hline $\mathrm{C}(1)-\mathrm{N}(1)-\mathrm{C}(2)$ & $122.8(5)$ & $\mathrm{C}(1)-\mathrm{N}(1)-\mathrm{C}(2)$ & $122.2(10)$ \\
\hline $\mathrm{C}(8)-\mathrm{N}(2)-\mathrm{C}(7)$ & $123.3(5)$ & $\mathrm{C}(8)-\mathrm{N}(2)-\mathrm{C}(7)$ & $124.2(10)$ \\
\hline $\mathrm{C}(8)-\mathrm{N}(2)-\mathrm{C}(9)$ & $121.8(5)$ & $\mathrm{C}(8)-\mathrm{N}(2)-\mathrm{C}(9)$ & $119.5(10)$ \\
\hline $\mathrm{N}(1)-\mathrm{C}(1)-\mathrm{S}(1)$ & $117.2(5)$ & $\mathrm{N}(1)-\mathrm{C}(1)-\mathrm{S}(1)$ & $118.5(9)$ \\
\hline$S(2)-C(1)-S(1)$ & $118.5(3)$ & $\mathrm{S}(2)-\mathrm{C}(1)-\mathrm{S}(1)$ & $118.8(8)$ \\
\hline $\mathrm{N}(2)-\mathrm{C}(8)-\mathrm{S}(3)$ & $118.0(4)$ & $\mathrm{N}(2)-\mathrm{C}(8)-\mathrm{S}(3)$ & $116.3(10)$ \\
\hline$S(4)-C(8)-S(3)$ & $118.4(4)$ & $S(4)-C(8)-S(3)$ & $119.0(8)$ \\
\hline \multicolumn{4}{|c|}{ Torsion Angles (deg) } \\
\hline $\mathrm{C}(24)-\mathrm{Sn}(1)-\mathrm{S}(1)-\mathrm{C}(1)$ & $78.4(3)$ & $\mathrm{C}(23)-\mathrm{Sn}(1)-\mathrm{S}(1)-\mathrm{C}(1)$ & $-73.2(6)$ \\
\hline $\mathrm{C}(23)-\mathrm{Sn}(1)-\mathrm{S}(1)-\mathrm{C}(1)$ & $-71.0(3)$ & $\mathrm{C}(27)-\mathrm{Sn}(1)-\mathrm{S}(1)-\mathrm{C}(1)$ & $78.6(5)$ \\
\hline$S(3)-S n(1)-S(1)-C(1)$ & $-177.26(19)$ & $S(3)-S n(1)-S(1)-C(1)$ & $-176.2(4)$ \\
\hline $\mathrm{S}(4)-\mathrm{Sn}(1)-\mathrm{S}(1)-\mathrm{C}(1)$ & $-175.03(19)$ & $\mathrm{S}(4)-\mathrm{Sn}(1)-\mathrm{S}(1)-\mathrm{C}(1)$ & $-175.9(4)$ \\
\hline $\mathrm{C}(24)-\mathrm{Sn}(1)-\mathrm{S}(2)-\mathrm{C}(1)$ & $-110.5(3)$ & $\mathrm{C}(23)-\mathrm{Sn}(1)-\mathrm{S}(2)-\mathrm{C}(1)$ & $103.4(5)$ \\
\hline $\mathrm{C}(23)-\mathrm{Sn}(1)-\mathrm{S}(2)-\mathrm{C}(1)$ & 109.7(3) & $\mathrm{C}(27)-\mathrm{Sn}(1)-\mathrm{S}(2)-\mathrm{C}(1)$ & $-117.3(5)$ \\
\hline$S(3)-S n(1)-S(2)-C(1)$ & $0.3(2)$ & $\mathrm{S}(3)-\mathrm{Sn}(1)-\mathrm{S}(2)-\mathrm{C}(1)$ & $-3.5(5)$ \\
\hline $\mathrm{S}(4)-\mathrm{Sn}(1)-\mathrm{S}(2)-\mathrm{C}(1)$ & $175.2(2)$ & $S(4)-S n(1)-S(2)-C(1)$ & $175.9(4)$ \\
\hline $\mathrm{C}(24)-\mathrm{Sn}(1)-\mathrm{S}(3)-\mathrm{C}(8)$ & $-80.7(3)$ & $\mathrm{C}(23)-\mathrm{Sn}(1)-\mathrm{S}(3)-\mathrm{C}(8)$ & $80.1(6)$ \\
\hline $\mathrm{C}(23)-\mathrm{Sn}(1)-\mathrm{S}(3)-\mathrm{C}(8)$ & $72.6(3)$ & $\mathrm{C}(27)-\mathrm{Sn}(1)-\mathrm{S}(3)-\mathrm{C}(8)$ & $-70.5(5)$ \\
\hline$S(1)-\operatorname{Sn}(1)-S(3)-C(8)$ & $176.30(19)$ & $\mathrm{S}(1)-\mathrm{Sn}(1)-\mathrm{S}(3)-\mathrm{C}(8)$ & $-178.4(4)$ \\
\hline $\mathrm{S}(2)-\mathrm{Sn}(1)-\mathrm{S}(3)-\mathrm{C}(8)$ & $174.50(19)$ & $\mathrm{S}(2)-\mathrm{Sn}(1)-\mathrm{S}(3)-\mathrm{C}(8)$ & $-178.6(4)$ \\
\hline $\mathrm{C}(24)-\mathrm{Sn}(1)-\mathrm{S}(4)-\mathrm{C}(8)$ & $111.6(3)$ & $\mathrm{C}(23)-\mathrm{Sn}(1)-\mathrm{S}(4)-\mathrm{C}(8)$ & $-109.5(6)$ \\
\hline$C(23)-S n(1)-S(4)-C(8)$ & $-109.6(3)$ & $\mathrm{C}(27)-\mathrm{Sn}(1)-\mathrm{S}(4)-\mathrm{C}(8)$ & $110.7(5)$ \\
\hline $\mathrm{S}(1)-\mathrm{Sn}(1)-\mathrm{S}(4)-\mathrm{C}(8)$ & $0.1(2)$ & $\mathrm{S}(1)-\mathrm{Sn}(1)-\mathrm{S}(4)-\mathrm{C}(8)$ & $-2.1(5)$ \\
\hline $\mathrm{S}(2)-\mathrm{Sn}(1)-\mathrm{S}(4)-\mathrm{C}(8)$ & $-174.4(2)$ & $\mathrm{S}(2)-\mathrm{Sn}(1)-\mathrm{S}(4)-\mathrm{C}(8)$ & $178.5(4)$ \\
\hline $\mathrm{C}(16)-\mathrm{N}(1)-\mathrm{C}(1)-\mathrm{S}(2)$ & $2.5(8)$ & $\mathrm{C}(16)-\mathrm{N}(1)-\mathrm{C}(1)-\mathrm{S}(2)$ & $0.9(15)$ \\
\hline $\mathrm{C}(2)-\mathrm{N}(1)-\mathrm{C}(1)-\mathrm{S}(1)$ & $3.2(7)$ & $\mathrm{C}(2)-\mathrm{N}(1)-\mathrm{C}(1)-\mathrm{S}(1)$ & $-0.6(14)$ \\
\hline $\mathrm{Sn}(1)-\mathrm{S}(2)-\mathrm{C}(1)-\mathrm{N}(1)$ & $-176.8(5)$ & $\operatorname{Sn}(1)-\mathrm{S}(2)-\mathrm{C}(1)-\mathrm{N}(1)$ & $-174.0(10)$ \\
\hline $\operatorname{Sn}(1)-\mathrm{S}(1)-\mathrm{C}(1)-\mathrm{N}(1)$ & $176.4(4)$ & $\operatorname{Sn}(1)-\mathrm{S}(1)-\mathrm{C}(1)-\mathrm{N}(1)$ & $173.2(9)$ \\
\hline $\mathrm{C}(9)-\mathrm{N}(2)-\mathrm{C}(8)-\mathrm{S}(4)$ & $2.8(8)$ & $\mathrm{C}(9)-\mathrm{N}(2)-\mathrm{C}(8)-\mathrm{S}(4)$ & $2.0(16)$ \\
\hline $\mathrm{C}(7)-\mathrm{N}(2)-\mathrm{C}(8)-\mathrm{S}(3)$ & $-1.8(7)$ & $\mathrm{C}(7)-\mathrm{N}(2)-\mathrm{C}(8)-\mathrm{S}(3)$ & $4.8(16)$ \\
\hline $\mathrm{Sn}(1)-\mathrm{S}(4)-\mathrm{C}(8)-\mathrm{N}(2)$ & $175.8(5)$ & $\operatorname{Sn}(1)-S(4)-C(8)-N(2)$ & $-173.9(11)$ \\
\hline $\mathrm{Sn}(1)-\mathrm{S}(3)-\mathrm{C}(8)-\mathrm{N}(2)$ & $-175.3(4)$ & $\mathrm{Sn}(1)-\mathrm{S}(3)-\mathrm{C}(8)-\mathrm{N}(2)$ & $173.8(9)$ \\
\hline
\end{tabular}




\section{Conclusions}

The results presented in this contribution have shown that the synthetic strategy previously applied to the formation of macrocyclic diorganotin carboxylates could be extended successfully to the preparation of dithiocarbamate analogues.

\section{Acknowledgements}

The authors thank CONACyT for financial support through project No. SEP-2004-C01-47347.

\section{Experimental Section}

General Procedures. All melting points were determined with a Yazawa micro melting point apparatus and are uncorrected. NMR spectra were recorded on Varian Gemini 200 and Varian Inova 400 instruments. Standards were TMS (internal ${ }^{1} \mathrm{H},{ }^{13} \mathrm{C}$ ) and $\mathrm{Me}_{4} \mathrm{Sn}$ (external, ${ }^{119} \mathrm{Sn}$ ). Chemical shifts are stated in parts per million; they are positive, when the signal is shifted to higher frequencies than the standart. COSY and HSQC experiments have been carried out in order to assign the ${ }^{1} \mathrm{H}$ and ${ }^{13} \mathrm{C}$ spectra completely. IR spectra have been recorded on a Bruker Vector $22 \mathrm{FT}$ spectrophotometer. The $\mathrm{FAB}^{+}$mass spectra were obtained on a Jeol JMS-700 equipment. Elemental Analyses have been carried out on a Perkin-Elmer series II 2400 instrument.

X-Ray crystallography. X-Ray diffraction studies of single crystals for compounds $\mathbf{1}$ and $\mathbf{2}$ were conducted on a BRUKER-AXS APEX diffractometer equipped with a CCD area detector ( $\lambda_{\mathrm{MoK} \alpha}=0.71073 \AA$, monochromator: graphite). Frames were collected via $\omega / \phi-$ rotation $(\Delta / \omega=$ $0.3^{\circ}$ ) at $10 \mathrm{~s}$ per frame (program SMART ${ }^{13}$ ). The measured intensities were reduced to $F^{2}$ and corrected for absorption with SADABS (program SAINT-NT ${ }^{14]}$ ), the cell parameters were determined by using reflections from all frames collected. Structure solution, refinement and data output were carried out with the SHELXTL-NT program package. ${ }^{15}$ Non hydrogen atoms were refined anisotropically. Hydrogen atoms were placed in geometrically calculated positions using a riding model.

Crystallographic data for the structures reported in this paper have been deposited with the Cambridge Crystallographic Data Centre as supplementary publication no. CCDC-650011650012. Copies of the data can be obtained free of charge on application to CCDC, 12 Union Road, Cambridge, CB2 1EZ, UK (fax: (+44) 1223-336-033, e-mail: deposit@ccdc.cam.ac.uk). 
Synthetic procedures. The preparation of the ligand required for the preparation of the diorganotin dithiocarbamates was carried out in two steps following procedures reported in the literature. $^{6}$

$\boldsymbol{N}, \boldsymbol{N}^{\prime}$-Dibenzylhexamethylene-1,6-diimine. 1,6-Hexamethylenediamine (1.00 g, $\left.8.6 \mathrm{mmol}\right)$ and two equivalents of benzaldehyde $(1.8 \mathrm{ml}, 18.5 \mathrm{mmol})$ were dissolved in ethanol $(25 \mathrm{~mL})$ and the solution was refluxed for $2 \mathrm{~h}$. After evaporation of the solvent the diimine was obtained in form of a yellow oil (yield:2.5 g, $98.5 \%$ ). IR $\left(\mathrm{KBr}, \mathrm{cm}^{-1}\right) v(\mathrm{C}=\mathrm{N}), 1645 ; v(\mathrm{C}-\mathrm{N}), 1218 ; v(\mathrm{C}-\mathrm{H}), 3060$; $v\left(\mathrm{C}-\mathrm{H}\right.$, out-of-plane), 753, $v$ (out-of-plane ring bend) $693 ;{ }^{1} \mathrm{H} \mathrm{NMR}\left(200 \mathrm{MHz}, \mathrm{CDCl}_{3}, \mathrm{ppm}\right) \delta$ $8.22(\mathrm{~s}, 2 \mathrm{H}, \mathrm{CH}=\mathrm{N}), 7.71-766\left(\mathrm{~m}, 4 \mathrm{H}, o-\mathrm{C}_{6} \mathrm{H}_{5}\right), 7.38-7.34\left(\mathrm{~m}, 6 \mathrm{H}, m, p-\mathrm{C}_{6} \mathrm{H}_{5}\right), 3.59(\mathrm{t}, 4 \mathrm{H}, J=$ $\left.6.0 \mathrm{~Hz}, \mathrm{NCH}_{2}\right), 1.71\left(\mathrm{~m}, 4 \mathrm{H}, \mathrm{NCH}_{2} \underline{\mathrm{CH}}_{2}\right), 1.41\left(\mathrm{~m}, 4 \mathrm{H}, \mathrm{NCH}_{2} \mathrm{CH}_{2} \underline{\mathrm{CH}}_{2}\right) ;{ }^{13} \mathrm{C} \mathrm{NMR}(200 \mathrm{MHz}$, $\left.\mathrm{CDCl}_{3}, \mathrm{ppm}\right) \delta 160.7(\mathrm{C}=\mathrm{N}), 136.1(\mathrm{Ci}), 130.3(\mathrm{Cp}), 128.5(\mathrm{Co}), 127.9(\mathrm{Cm}), 61.7\left(\mathrm{NCH}_{2} \underline{\mathrm{CH}}_{2}\right)$, $31.1\left(\mathrm{NCH}_{2} \underline{\mathrm{CH}_{2}}\right), 27.4\left(\mathrm{NCH}_{2} \mathrm{CH}_{2} \underline{\mathrm{CH}_{2}}\right)$.

$\boldsymbol{N}, \boldsymbol{N}^{\prime}$-dibenzylhexamethylene-1,6-diamine. $N, N^{\prime}$-dibenzylhexamethylene-1,6-diimine (2.51 g, $8.6 \mathrm{mmol}$ ) was dissolved in methanol and the solution was cooled to $0 \mathrm{C}^{\circ}$. Sodium borohydride ( $0.97 \mathrm{~g}, 25.8 \mathrm{mmol})$ was added under stirring and the mixture was allowed to react over night. After removal of the solvent under vacuum, the resulting viscous liquid was washed with water and dichloromethane was added in order to extract the product. The organic layer was dried over anhydrous $\mathrm{MgSO}_{4}$. After filtration the solvent was evaporated to obtain a yellow oil that was identified as the product (yield: $2.44 \mathrm{~g}, 96 \%$ ). IR $\left(\mathrm{KBr}, \mathrm{cm}^{-1}\right) v(\mathrm{~N}-\mathrm{H}) 3385$; $v(\mathrm{C}-\mathrm{N}), 1218,1050$; $v\left(\mathrm{C}-\mathrm{H}\right.$, out-of-plane), 736; $v$ (out-of-plane ring bend) $698 ;{ }^{1} \mathrm{H} \mathrm{NMR}\left(400 \mathrm{MHz}, \mathrm{CDCl}_{3}, \mathrm{ppm}\right) \delta$ $7.30\left(\mathrm{~m}, 10 \mathrm{H},-\underline{\mathrm{C}}_{6} \underline{\mathrm{H}}_{5}\right), 3.76\left(\mathrm{~s}, 4 \mathrm{H}, \underline{\mathrm{NCH}}_{2}-\mathrm{C}_{6} \mathrm{H}_{5}\right), 2.60$ (t, $\left.4 \mathrm{H}, \mathrm{t}, J=7.6 \mathrm{~Hz}, \underline{\mathrm{NCH}}_{2}\right), 1.73$ (br, 2H, $\mathrm{N}-\mathrm{H}), 1.49\left(\mathrm{~m}, 4 \mathrm{H}, \mathrm{NCH}_{2} \underline{\mathrm{CH}}_{2}\right), 1.31\left(\mathrm{~m}, 4 \mathrm{H}, \mathrm{NCH}_{2} \mathrm{CH}_{2} \underline{\mathrm{CH}}_{2}\right) ;{ }^{13} \mathrm{C} \mathrm{NMR}\left(400 \mathrm{MHz}, \mathrm{CDCl}_{3}\right.$, ppm) $\delta 140.5(\mathrm{Ci}), 128.5(\mathrm{Co}), 128.2(\mathrm{Cm}), 127.0(\mathrm{Cp}), 54.2\left(\mathrm{NCH}_{2} \mathrm{C}_{6} \mathrm{H}_{5}\right), 49.6\left(\mathrm{NCH}_{2}\right)_{2} 30.2$ $\left(\mathrm{NCH}_{2} \underline{\mathrm{CH}}_{2}\right), 27.5\left(\mathrm{NCH}_{2} \mathrm{CH}_{2} \underline{\mathrm{CH}}_{2}\right) ; \mathrm{MS} \mathrm{m} / z: 296\left(\mathrm{M}^{+}, 10 \%\right)$.

$\left[\mathbf{M e}_{2} \operatorname{Sn}(\mathbf{d t c})\right]_{2} \quad \mathbf{( 1 )} . \quad N, N^{\prime}$-dibenzylhexamethylene-1,6-diamine $\quad(0.05 \quad \mathrm{~g}, \quad 0.168 \mathrm{mmol})$, triethylamine $(0.047 \mathrm{~mL}, 0.33 \mathrm{mmol})$ and carbon disulfide $(0.5 \mathrm{~mL}, 8 \mathrm{mmol})$ were dissolved in methanol $(5 \mathrm{~mL})$ and stirred for $2 \mathrm{~h}$. $\mathrm{Me}_{2} \mathrm{SnCl}_{2}(0.037 \mathrm{~g}, 0.168 \mathrm{mmol}$,) was dissolved in $3 \mathrm{ml}$ of methanol and added to the solution. A white powder precipitated that was filtered and recrystallized from chloroform (yield: $0.10 \mathrm{~g}, 54 \%$ ), mp 150-153 ${ }^{\circ} \mathrm{C}$. IR $\left(\mathrm{KBr}, \mathrm{cm}^{-1}\right) v(\mathrm{C}-\mathrm{N})$, 1476; $v\left(\mathrm{CS}_{2}\right)_{\text {as }}, 1239 ; v\left(\mathrm{CS}_{2}\right)_{\mathrm{s}}, 981 ; v(\mathrm{C}-\mathrm{N}), 1123$; $v(\mathrm{C}-\mathrm{H}$, out-of-plane), 733; v(out-of-plane ring bend) 698; ${ }^{1} \mathrm{H}$ NMR (400 MHz, $\left.\mathrm{CDCl}_{3}, \mathrm{ppm}\right) \delta 7.36-7.26\left(\mathrm{~m}, 20 \mathrm{H}, 4 \underline{\mathrm{C}}_{6} \underline{\mathrm{H}}_{5}\right), 5.15$ (s, 8H, 4 $\underline{\mathrm{CH}_{2}} \mathrm{C}_{6} \mathrm{H}_{5}$ ), 3.62 (br, 8H, $4 \underline{\mathrm{NCH}}_{2}$ ), 1.68 (br, 8H, $4 \mathrm{NCH}_{2} \underline{\mathrm{CH}}_{2}$ ), 1.60 (s, 12H, $4 \mathrm{SnCH}_{3}$ ), 1.22 (br, $\left.8 \mathrm{H}, 4 \mathrm{NCH}_{2} \mathrm{CH}_{2} \mathrm{CH}_{2}\right) ;{ }^{13} \mathrm{C} \mathrm{NMR}\left(400 \mathrm{MHz}, \mathrm{CDCl}_{3}, \mathrm{ppm}\right) \delta 201.0\left(\mathrm{CS}_{2}\right), 135.7\left(\mathrm{C}_{i}\right), 128.8(\mathrm{Co})$, $127.7\left(\mathrm{C}_{m}\right), 127.9\left(\mathrm{C}_{p}\right), 57.5\left(\underline{\mathrm{CH}}_{2} \mathrm{C}_{6} \mathrm{H}_{5}\right), 54.0\left(\mathrm{NCH}_{2}\right), 27.3\left(\mathrm{NCH}_{2} \underline{\mathrm{CH}}_{2}\right), 27.1\left(\mathrm{NCH}_{2} \mathrm{CH}_{2} \underline{\mathrm{CH}}_{2}\right)$; $15.5\left(\mathrm{SnCH}_{3},{ }^{2} J_{\mathrm{Sn}-\mathrm{H}}=82 \mathrm{~Hz}\right),{ }^{119} \mathrm{Sn} \mathrm{RMN}\left(200 \mathrm{MHz}, \mathrm{CDCl}_{3}, \mathrm{ppm}\right) \delta-332(\mathrm{Sn}) ; \mathrm{FAB}-\mathrm{MS} m / z$ : $1190\left(\mathrm{M}^{+}, 2\right)$. Anal. Calcd (\%) for $\mathrm{C}_{48} \mathrm{H}_{64} \mathrm{~N}_{4} \mathrm{~S}_{8} \mathrm{Sn}_{2} \cdot 2 \mathrm{CHCl}_{3}\left(1429\right.$ gmol $\left.^{-1}\right): \mathrm{C}, 42.00 ; \mathrm{H}, 4.65 ; \mathrm{N}$, 3.91. Found: C, 41.32; H, 4.54; N, 4.22. 
$\left[\boldsymbol{n} \mathbf{B u}_{2} \mathbf{S n}(\mathbf{d t c})\right]_{2} \quad$ (2). $\quad N, N^{\prime}$-dibenzylhexamethylene-1,6-diamine $\quad(0.05 \quad \mathrm{~g}, \quad 0.168 \mathrm{mmol})$, triethylamine $(0.047 \mathrm{~mL}, 0.33 \mathrm{mmol})$ and carbon disulfide $(0.5 \mathrm{~mL})$ were dissolved in methanol $(5 \mathrm{~mL})$ and stirred for $2 \mathrm{~h} . n \mathrm{Bu}_{2} \mathrm{SnCl}_{2}(0.05 \mathrm{~g}, 0.168 \mathrm{mmol})$ was dissolved in $3 \mathrm{ml}$ of methanol and added to the solution. A precipitate formed that was filtered and recrystallized from a mixture of dichloromethan/methanol $(0.08 \mathrm{~g}, 36 \%)$, mp 173-175 ${ }^{\circ} \mathrm{C}$. IR $\left(\mathrm{KBr}, \mathrm{cm}^{-1}\right) v_{\mathrm{C}-\mathrm{N}}, v(\mathrm{C}-$ $\mathrm{N}), 1475 ; v\left(\mathrm{CS}_{2}\right)_{\text {as }}, 1238, v\left(\mathrm{CS}_{2}\right)_{\mathrm{s}}, 980 ; v(\mathrm{C}-\mathrm{N}), 1120 ; v(\mathrm{C}-\mathrm{H}$, out-of-plane), $732 ; v$ (out-of-plane ring bend) 699; ${ }^{1} \mathrm{H} \mathrm{NMR}\left(400 \mathrm{MHz}, \mathrm{CDCl}_{3}, \mathrm{ppm}\right) \delta{ }^{1} \mathrm{H} \mathrm{NMR}\left(400 \mathrm{MHz}, \mathrm{CDCl}_{3}, \mathrm{ppm}\right) \delta 7.36-$ $7.26\left(\mathrm{~m}, 20 \mathrm{H}, 5 \mathrm{C}_{6} \mathrm{H}_{5}\right), 5.18\left(\mathrm{~s}, 8 \mathrm{H}, 4 \underline{\mathrm{CH}}_{2} \mathrm{C}_{6} \mathrm{H}_{5}\right), 3.72-3.62\left(\mathrm{~m}, 8 \mathrm{H}, 4 \mathrm{NCH}_{2}\right), 2.17-2.11(\mathrm{~m}, 8 \mathrm{H}$, $4 \underline{\mathrm{CH}}_{2} \mathrm{Sn}$ ), 2.0-1.95 (m, 8H, $4 \underline{\mathrm{CH}}_{2} \mathrm{CH}_{2} \mathrm{Sn}$ ), 1.69 (br, 8H, $4 \mathrm{NCH}_{2} \underline{\mathrm{CH}}_{2}$ ), 1.51-1.42 (m, 8H, 4 $\underline{\mathrm{CH}_{2}} \mathrm{CH}_{2} \mathrm{CH}_{2} \mathrm{Sn}$ ), 1.23 (br, 8H, $4 \mathrm{NCH}_{2} \mathrm{CH}_{2} \underline{\mathrm{CH}}_{2}$ ), 0.95 (t, J=7.2 Hz, $12 \mathrm{H}, 4 \underline{\mathrm{CH}}_{3} \mathrm{CH}_{2} \mathrm{CH}_{2} \mathrm{CH}_{2} \mathrm{Sn}$ ); ${ }^{13} \mathrm{C}$ NMR (400 MHz, $\left.\mathrm{CDCl}_{3}, \mathrm{ppm}\right) \delta 201.8\left(\mathrm{CS}_{2}\right), 135.8\left(\mathrm{C}_{i}\right), 128.9(\mathrm{Co}), 127.6(\mathrm{Cm}), 127.9$ $\left(\mathrm{C}_{p}\right), 57.5\left(\underline{\mathrm{CH}_{2}} \mathrm{C}_{6} \mathrm{H}_{5}\right), 54.2\left(\underline{\mathrm{NCH}}_{2}\right), 27.4\left(\mathrm{NCH}_{2} \underline{\mathrm{CH}}_{2}\right), 34.2\left(\underline{\mathrm{CH}_{2}} \mathrm{Sn}\right), 28.9\left(\underline{\mathrm{CH}}_{2} \mathrm{CH}_{2} \mathrm{Sn}\right), 27.1$ $\left(\mathrm{NCH}_{2} \mathrm{CH}_{2} \underline{\mathrm{CH}_{2}}\right), 26.8\left(\underline{\mathrm{CH}_{2}} \mathrm{CH}_{2} \mathrm{CH}_{2} \mathrm{Sn}\right), 14.2\left(\underline{\mathrm{CH}}_{3} \mathrm{CH}_{2} \mathrm{CH}_{2} \mathrm{CH}_{2} \mathrm{Sn}\right) ;{ }^{119} \mathrm{Sn} \mathrm{RMN}(200 \mathrm{MHz}$, $\mathrm{CDCl}_{3}$, ppm) $\delta$-333; FAB-MS m/z: 1066 (M-(benzyl)N $\left(\mathrm{CH}_{2}\right)_{6} \mathrm{~N}($ benzyl), 30), 947 (M-dtc+S, 34). Anal. Calcd (\%) for $\mathrm{C}_{60} \mathrm{H}_{88} \mathrm{~N}_{4} \mathrm{~S}_{8} \mathrm{Sn}_{2}\left(1359.21 \mathrm{gmol}^{-1}\right)$ : C, 52.9; H, 6.47; N, 4.12. Found: C, $52.45 ; \mathrm{H}, 6.46 ; \mathrm{N}, 4.58$.

\section{References}

1. For reviews see: (a) Langley, P. J.; Hulliger, J. Chem. Soc. Rev. 1999, 28, 279. (b) Swiegers, G. F.; Malefetse, T. J. Chem. Rev. 2000, 100, 3483. (c) Moulton, B.; Zaworotko, M. J. Chem. Rev. 2001, 101, 1629. (d) Würthner, F.; You, C.-C.; Saha-Möller, C. R. Chem. Soc. Rev. 2004, 33, 133.

2. For macrocyclic or porous systems containing main group elements other than tin see: (a) Robl, C.; Weiss, A. Z. Naturforsch. B 1986, 41, 1485. (b) Robl, C.; Weiss A. Z. Naturforsch. $B$ 1986, 41, 1490. (c) Lee, C. R.; Wang, C. C.; Wang, Y. Acta Cryst. B 1996, 52, 966. (d) Román, P.; Miralles, C. G. ; Luque, A. J. Chem. Soc., Dalton Trans. 1996, 3985. (e) Plater, M. J.; Roberts, A. J.; Marr, J.; Lachowski, E. E.; Howie, R. A. J. Chem. Soc. Dalton Trans. 1998, 797. (f) Rettig, S. J.; Storr, A.; Trotter, J. Can. J. Chem. 1999, 77, 434. (g) Lightfoot, P.; Lethbridge, Z. A. D.; Morris, R. E.; Wragg, D. S.; Wright, P. A.; Kvick, A.; Vaughan, G. B. M. J. Solid State Chem. 1999, 143, 74. (h) Chen, C. Y.; Chu, P. P.; Lii, K. H. Chem. Commun. 1999, 1473. (i) Kedarnath, K.; Choudhury, A.; Natarajan, S. J. Solid State Chem. 2000, 150, 324. (j) Hung, L. C.; Kao, H. M.; Lii, K. H. Chem. Mater. 2000, 12, 2411. (k) Uhl, W. Chem. Soc. Rev. 2000, 29, 259. (1) Choi, C. T. S.; Anokhina, E. V.; Day, C. S.; Zhao, Y.; Taulelle, F.; Huguenard, C.; Gan, Z.; Lachgar, A. Chem. Mater. 2002, 14, 4096. (m) Huang, Y. F.; Lii, K. H. J. Chem. Soc., Dalton Trans. 1998, 4085.

3. For macrocyclic or porous systems containing tin see: (a) Lockhart, T. P. Organometallics 1988, 7, 1438. (b) Ng, S. W.; Das, V. G. K.; Pelizzi, G.; Vitali, F. Heteroatom Chem. 1990, 1, 433. (c) Meunier-Piret, J.; Boualam, M.; Willem, R.; Gielen M. Main Group Met. Chem. 
1993, 16, 329. (d) Gielen, M.; Khloufi, A. E.; Biesemans, M.; Kayser, F.; Willem, R. Organometallics 1994, 13, 2849. (e) Gajda-Schranz, K. ; Nagy, L.; Kuzmann, E.; Vértes A.; Holecek, J.; Lycka, A. J. Chem. Soc., Dalton Trans. 1997, 2201. (f) Natarajan, S.; Vaidhyanathan, R.; Rao, C. N. R.; Ayyappan, S.; Cheetham, A. K. Chem. Mater. 1999, 11, 1633. (g) Salami, T. O.; Zavilij, P. Y.; Oliver, S. R. J. Acta Cryst. E 2001, 57, m111. (h) Natarajan, S. J. Solid State Chem. 1998, 139, 200. (i) Ma, C.; Jiang, Q.; Zhang, R.; Wang, D. J. Chem. Soc., Dalton Trans. 2003, 2975. (j) Ma, C.; Jiang, Q.; Zhang, R. J. Organomet. Chem. 2003, 678, 148. (k) García-Zarracino, R.; Ramos-Quiñones, J.; Höpfl, H. Inorg. Chem. 2003, 42, 3835.

4. (a)Webber, P. R. A.; Drew, M. G. B.; Hibbert, R.; Beer, P. D. J. Chem. Soc., Dalton Trans. 2004, 8, 1127. (b) Fox, O. D.; Drew, M. G. B.; Beer, P. D. Angew. Chem. Int. Ed. 2000, 39, 136. (c) Padilla-Tosta, M. E.; Fox, O. D.; Drew, M. G. B.; Beer, P. D. Angew. Chem. Int. Ed. 2001, 40, 4235. (d) Beer, P. D.; Berry, N.; Drew, M. G. B.; Fox, O. D.; Padilla-Tosta, M. E.; Patell, S. Chem. Commun. 2001, 199. (e) Berry, N.G.; Pratt, M. D.; Fox, O. D.; Beer, P. D. Supramol. Chem. 2001, 13, 677. (f) Beer, P. D.; Cheetham, A. G.; Drew, M. G. B.; Fox, O. D.; Hayes, E. J.; Rolls, T. D. J. Chem. Soc., Dalton Trans. 2003, 4, 603. (g) Chandrasekhar, V.; Day, R.O.; Holmes, J.M.; Holmes, R. R. Inorg. Chem. 1988, 27, 958.

5. (a) García-Zarracino, R.; Ramos-Quiñones, J.; Höpfl, H. Inorg. Chem. 2003, 42, 3835. (b) García-Zarracino, R.; Höpfl, H. Angew. Chem. Int. Ed. 2004, 43, 1507. (c) García-Zarracino, R.; Höpfl, H. Appl. Organomet. Chem. 2005, 19, 451. (d) García-Zarracino, R.; Höpfl, H. J. Am. Chem. Soc. 2005, 127, 3120.

6. Wong, W. W. E; Curiel, D.; Lai, S.-W.; Drew, M. G. B.; Beer, P. D. J. Chem. Soc., Dalton Trans. 2005, 774.

7. (a) Yin, H. D; Wang, C. H; Ma, C. L; Wang, Y; Zhang, R. F. Chin. J. Inorg. Chem. 2002, 18, 347. (b) Yin, H. D; Wang, C. H; Ma, C. L; Wang, Y; Zhang, R. F. Chin. J. Inorg. Chem. 2002, 22, 183.

8. Ronconi, L; Giovagnini, L; Marzano, C; Bettio, F.; Graziani, R.; Pilloni, G.; Fregona, D. Inorg. Chem. 2005, 44, 1867.

9. (a) Dakternieks, D; Zhu, H; Masi, D; Mealli, C. Inorg. Chem. 1992, 31, 3601. (b) Yin, H. D; Xue, S. C. Appl. Organometal. Chem. 2006, 20, 283.

10. Lehn, J.-M. Angew. Chem. Int. Ed. 1990, 29, 1304.

11. (a) Desiraju, G. R. Acc. Chem. Res. 1996, 29, 441. (b) Desiraju, G. R. Acc. Chem. Res. 1991, 24, 290. (c) Sarma, J. A. R. P.; Desiraju, G. R. Acc. Chem. Res. 1986, 19, 222.

12. (a) Drago, R. S.; Structure Bonding 1973, 15, 73. (b) Jonas, V.; Frenking, G.; Reetz, M. T. J. Am. Chem. Soc. 1994, 116, 8741. (c) García-Hernández, Z.; Flores-Parra, A.; Grevy, J. M.; Ramos-Organillo, A; Contreras, R. Polyhedron 2006, 25, 1662.

13. Bruker Analytical X-ray Systems. SMART: Bruker Molecular Analysis Research Tool V. 5 .6182000 .

14. Bruker Analytical X-ray Systems. SAINT + NT Version 6.04 2001.

15. Bruker Analytical X ray Systems. SHELXTL-NT Version 6.10 2000. 